\title{
A case report of intracholecystic papillary neoplasm of the gallbladder resembling a submucosal tumor
}

\author{
Ryo Muranushi ${ }^{1 *}$, Hideyuki Saito ${ }^{2}$, Asuka Matsumoto $^{2}$, Toshihide Kato ${ }^{2}$, Naritaka Tanaka², Kenji Nakazato², \\ Nobuhiro Morinaga², Yoshinori Shitara ${ }^{2}$, Masatoshi Ishizaki ${ }^{2}$, Takatomo Yoshida ${ }^{3}$, Shinichi Aishima ${ }^{4}$ \\ and Ken Shirabe
}

\begin{abstract}
Background: Intracholecystic papillary neoplasm (ICPN) is defined as papillary tumors detected macroscopically in the gallbladder. We report a case of ICPN which exhibited the atypical form like a submucosal tumor.

Case presentation: A 70-year-old man was admitted to our hospital because of hepatic disorder. Computed tomography and magnetic resonance imaging showed irregular thickening of the wall within the gallbladder fundus. Because the lesion might have been malignant, we performed laparoscopic cholecystectomy and liver bed resection. Macroscopic findings showed the mucosal surface of the tumor was smooth, and its form was similar to that of a submucosal tumor. Histopathological examination revealed papillary tumors within the mass with low-grade dysplasia; therefore, we diagnosed ICPN.

Conclusion: In the present case, ICPN was resembling a submucosal tumor macroscopically because the tumors arose into the Rokitansky-Aschoff sinus and the adenomyomatous hyperplasia was merged with the ICPN. It is necessary to consider the possibility of tumor lesions within adenomyomatous hyperplasia.
\end{abstract}

Keywords: Intracholecystic papillary neoplasm, Adenomyomatous hyperplasia, Laparoscopic cholecystectomy

\section{Background}

Intracholecystic papillary neoplasm (ICPN) is preinvasive neoplastic lesions characterized by papillary growth in the gallbladder. ICPN is defined as gallbladder lesions of intraductal papillary neoplasm of the bile duct (IPNB). IPNB is a premalignant lesion of the biliary tract and is counterpart of intraductal papillary-mucinous neoplasm (IPMN) in the pancreatic duct epithelium [1]. ICPN is a papillary tumor generally detected macroscopically and is sometimes diagnosed by imaging findings. Herein, we report a case of ICPN which exhibited atypical form and which was distinguished difficultly from gallbladder adenocarcinoma.

Through this case, we consider clinicopathological characteristics and therapeutic strategies of ICPN.

\footnotetext{
* Correspondence: rmuranushi@gunma-u.ac.jp

${ }^{1}$ Department of Hepatobiliary and Pancreatic Surgery, Gunma University Graduate School of Medicine, Gunma University, 3-39-15 Showa-Machi, Maebashi, Gunma 371-8511, Japan

Full list of author information is available at the end of the article
}

\section{Case presentation}

The patient was a 70-year-old man. He was admitted to our hospital because of a hepatic disorder that was discovered during a routine health examination. Blood tests showed aspartate aminotransferase $48 \mathrm{U} / \mathrm{L}$ (normal range, 13 to $33 \mathrm{U} / \mathrm{L}$ ), alanine phosphatase $66 \mathrm{U} / \mathrm{L}$ (normal range, 8.0 to $42 \mathrm{U} / \mathrm{L}$ ), alkaline phosphatase $263 \mathrm{U} / \mathrm{L}$ (normal range, 115 to $359 \mathrm{U} / \mathrm{L}$ ), gamma-glutamyl transpeptidase 100 (normal range, 10 to $47 \mathrm{IU} / \mathrm{L}$ ), total bilirubin $0.5 \mathrm{mg} / \mathrm{dL}$ (normal range, 0.2 to $1.2 \mathrm{mg} / \mathrm{dL}$ ), carcinoembryonic antigen $4.4 \mathrm{ng} / \mathrm{mL}$ (normal range, < $5.0 \mathrm{ng} / \mathrm{ml}$ ), and carbohydrate antigen $19-910.4 \mathrm{U} / \mathrm{mL}$ (normal range, $<15 \mathrm{U} / \mathrm{mL}$ ). Abdominal ultrasonography showed an $8 \times 7$ - $\mathrm{mm}$ solid mass at the gallbladder fundus and several stones in the gallbladder (Fig. 1a). Enhanced computed tomography (CT) showed that irregular wall thickening at the gallbladder fundus and the boundary between tumor and the liver was indistinct (Fig. 1b). T2-weighted magnetic resonance imaging 


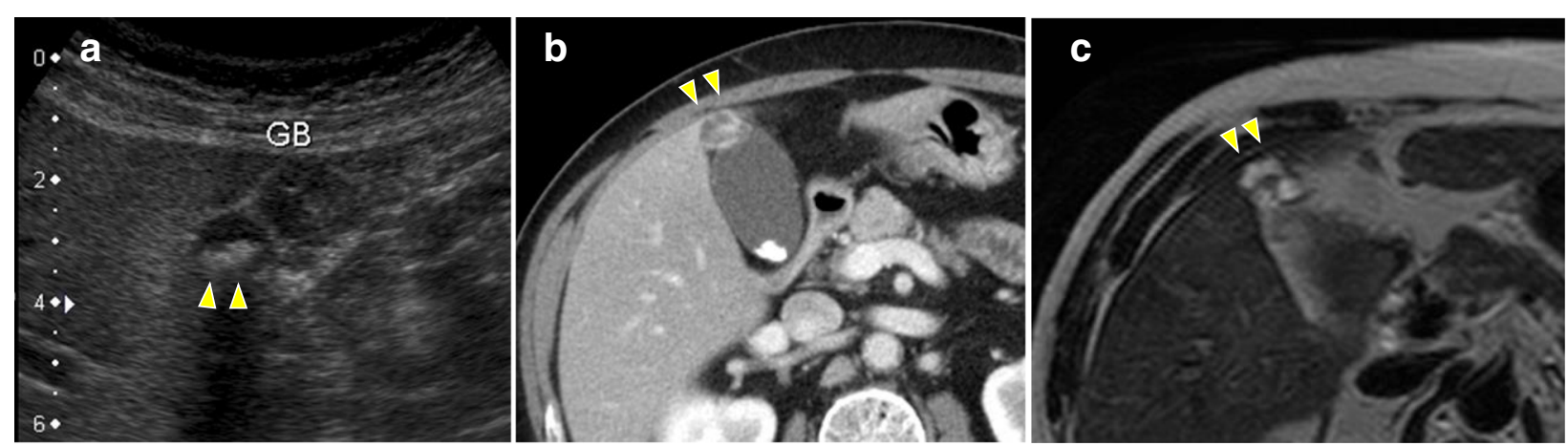

Fig. 1 Preoperative imaging findings. a Abdominal ultrasonography showed an $8 \times 7-\mathrm{mm}$ solid mass at the gallbladder fundus. $\mathbf{b}$ Enhanced CT showed that irregular wall thickening at the gallbladder fundus, and the boundary between the tumor and liver was indistinct. c T2-weighted MRI showed high-intensity nodules inside the thickened wall of the gallbladder

(MRI) showed a high-intensity nodule inside the thickened wall at the gallbladder fundus (Fig. 1c). According to these findings, we diagnosed the lesion as suspicious of malignancy and decided to perform surgery. During surgery, a tumor of approximately $10 \mathrm{~mm}$ was found at the gallbladder fundus and color change of the liver bed floor adjacent to the tumor was detected. We performed laparoscopic cholecystectomy and liver bed resection. The macroscopic findings of the resected specimen showed a $15 \times 10$-mm milky yellow mass at the gallbladder fundus, and its cut surface showed papillary lesions (Fig. 2). The tumor mucosal surface was smooth, and its form was similar to that of a submucosal tumor. Histopathological findings showed papillary tumors with cyst formation, and the tumors represented mucin secretion (Fig. 3a). Additionally, the Rokitansky-Aschoff sinus (RAS) was formed, and the smooth muscle became hyperplasia in the stromal tissue surrounding the papillary tumors (Fig. 3a, b). Within the epithelial cells, nucleus chromatin increased, karyotype was irregular, and nuclear body became clear. There was no invasion into the stromal tissue. These findings demonstrated ICPN with low-grade dysplasia (Fig. 3c). There was neither dysplasia nor biliary intraepithelial neoplasia on the background mucosa. Immunohistochemical analysis of the mucosal characteristics showed that MUC1, MUC5AC, and MUC6 were positive, whereas MUC2 was negative (Fig. 4). According to the predominant pattern on morphology and the mucin expression form, it was diagnosed as biliary type. Additionally, because Ki67 index was a little less than $10 \%$, it was denied that the tumor was malignant. The postoperative course was good, and the patient was discharged 9 days after the operation. A recurrence has not been detected for 3.5 years.

\section{Discussion}

ICPN was first described as gallbladder lesions of IPNB in the 2010 WHO classification and was classified as premalignant lesions of biliary system in the same category as adenoma, biliary intraepithelial neoplasia, and mucinous cystic neoplasm [2]. IPNB is defined as biliary tumors with an exophytic nature exhibiting papillary mass which can be detected macroscopically within the
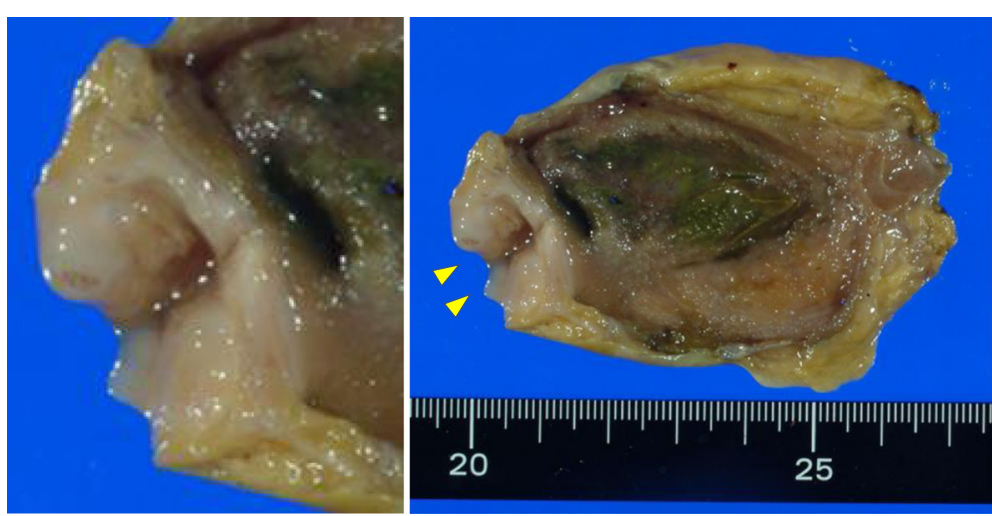

Fig. 2 Photograph of resected specimen. It shows the gallbladder fundus on the left side and the cystic duct on the right side. A $15 \times 10-\mathrm{mm}$ mass like a submucosal tumor is visible within the gallbladder fundus (arrow), and its cut surface shows the papillary lesions 


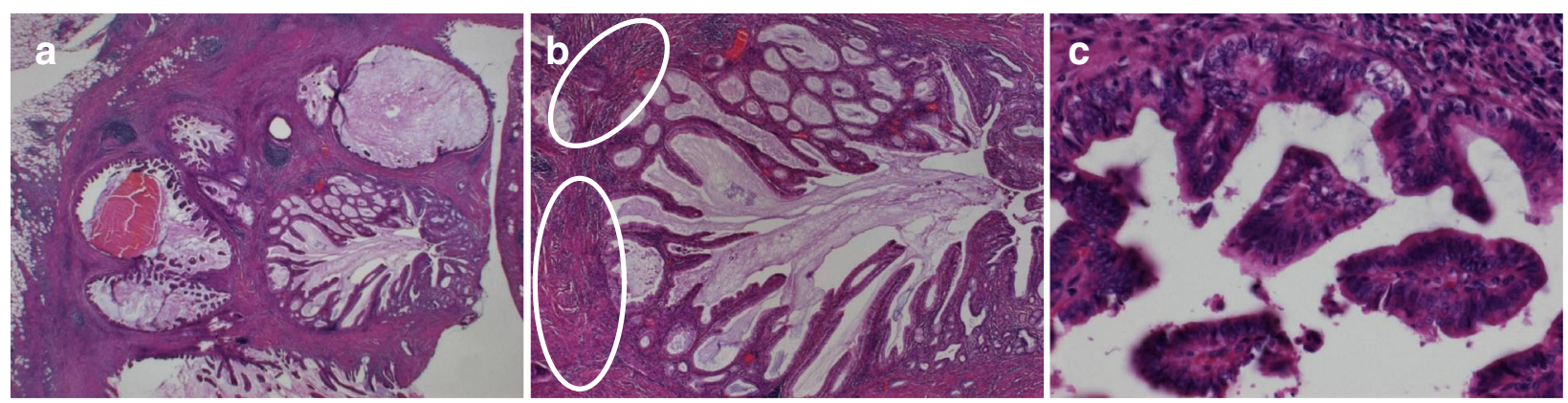

Fig. 3 Histopathological findings. a Papillary tumors with cyst formation which presented mucin secretion were detected, and the RokitanskyAschoff sinus was formed (hematoxylin-eosin staining, $\times 40$ ). $\mathbf{b}$ The smooth muscle became hyperplastic in the stromal tissue surrounding the papillary tumors (circle) (hematoxylin-eosin staining, $\times 100$ ). b Epithelial cells. The nucleus chromatin increased, the karyotype was irregular, and the nuclear body became clear, showing low-grade dysplasia. (Hematoxylin-eosin staining, $\times 400$ )

bile duct lumen, characterized by intraluminal growth [3]. It is associated with mucobilia due to excessive mucin secretion and is more commonly found in the hepatic biliary system [4].

The histopathological characteristics of ICPN are equivalent to IPNB. Four histological subtypes exist, including biliary type, gastric type, intestinal type, and oncocytic type according to IPMN. The mucus glycoprotein expression form is different depending on each histological subtype; that of early biliary carcinoma differs from ICPN $[5,6]$. About biliary type, Adsay et al. reported $68 \%$ of those represented cancer in situ and $69 \%$ included infiltrating cancer [7]. Correlation between mucin expression form and prognosis about several tumors has been studied. The current study showed that MUC1 immunohistochemical staining is a poor prognostic marker for IPNB [5]. Although there are few reports on ICPN, it is considered that this case should be followed closely.

ICPN shows various degrees of dysplasia from low- to high-grade and finally to invasive carcinoma, and the histological findings are often mixed [4]. This variation of dysplastic degree demonstrates the adenoma-carcinoma sequence while papillary adenocarcinoma is assumed to arise through de novo carcinogenesis. Therefore, from the perspective of carcinogenesis, ICPN is distinguished from
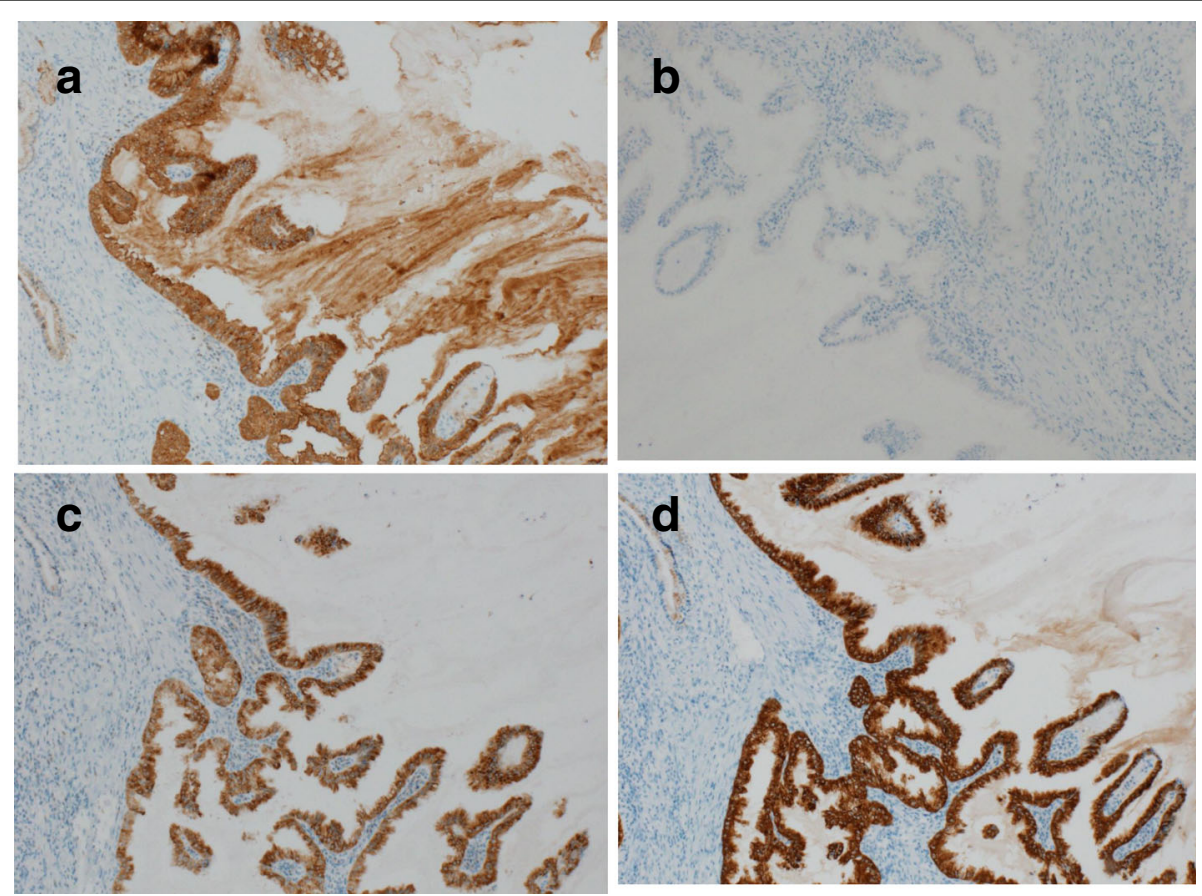

Fig. 4 Immunohistochemical analysis of mucosal characteristics. a MUC1 was positive; b MUC2 was negative; c MUC5AC was positive; and $\mathbf{d}$ MUC6 was positive 
papillary adenocarcinoma and it is related to the difference of each tumor prognosis. ICPN rarely infiltrates and metastasizes, and the prognosis for ICPN is typically much better than that for gallbladder adenocarcinoma. The 5 -year survival rate for ICPN is $60 \%$ if including invasive carcinoma and $78 \%$ if excluding the invasive region. In contrast, the 5-year survival rate for gallbladder adenocarcinoma is 30\% [7]. Therefore, it is important to diagnose ICPN correctly.

However, the concept of ICPN remains unclear about several points.

First, it is difficult to distinguish ICPN, including widespread infiltrating cancerous regions, from gallbladder carcinoma, including papillary tumor regions. It is important to distinguish both for selecting an appropriate treatment strategy. If ICPN is diagnosed preoperatively, simple cholecystectomy can be performed without lymphadenectomy. Second, management for tubular components in ICPN is controversial. There is no clear description about it in the 2010 WHO classification. Adsay et al. defines ICPN as an exophytic intramucosal gallbladder mass greater than $1 \mathrm{~cm}$ and composed of dysplastic cells forming a lesion distinct from the neighboring mucosa [7]. According to their definition, the existence of tubular components is not important if the condition mentioned above is satisfied. Additionally, it is considered that macroscopic papillary lesions are not essential for diagnosis.

In the present case, the tumor's mucosal surface was smooth and its form was similar to a submucosal tumor. Although the macroscopic findings were atypical, histopathological examination showed papillary tumors with low-grade dysplasia and mucin secretion and ICPN was diagnosed. The RAS was formed, the smooth muscle became hyperplastic in the stromal tissue, the tumors arose into the RAS, and the adenomyomatous hyperplasia was merged with ICPN. Thus, the tumor formed like a submucosal tumor.

Although histopathological findings showed papillary tumors with cystic formation, preoperative CT showed only wall thickening and did not show cystic formation findings. It was considered that because the cysts were involved in hyperplastic smooth muscles, the imaging could not detect the cystic formation. On the other hand, the high-intensity punctiform lesions were detected by T2-weighted MRI. It was considered that the finding demonstrated the tumors rise into the RAS, and the cysts were formed.

Although gallbladder adenocarcinoma or ICPN rising in RAS have been reported, to our knowledge, this is the first case of ICPN with adenomyomatous hyperplasia [8]. It is necessary to consider the possibility of tumor lesions within adenomyomatous hyperplasia. As in this case, coexistence of fundal-type adenomyomatous hyperplasia makes diagnosis difficult. In cases of adenomyomatous hyperplasia with irregular wall thickening and sequential changes, it is necessary to follow closely about the merger of the neoplastic lesion.

\section{Conclusion}

We report a case of ICPN that was resembling a submucosal tumor because the tumors rose into the RAS, and adenomyomatous hyperplasia was merged with ICPN. It is necessary to consider the possibility of tumor lesions within adenomyomatous hyperplasia.

\section{Abbreviations \\ ICPN: Intracholecystic papillary neoplasm; IPNB: Intraductal papillary neoplasm of the bile duct; RAS: Rokitansky-Aschoff sinus}

\section{Authors' contributions}

RM reported the case and wrote the manuscript. HS, AM, TK, NT, KN, NM, YS, and $\mathrm{MI}$ were involved in treating the patient. TY and SI diagnosed the pathological findings. SI and KS participated in critically revising the manuscript. All authors have read and approved the final manuscript.

Ethics approval and consent to participate

Not applicable.

\section{Consent for publication}

Written informed consent was obtained from the patient for the publication of this case report and the accompanying images.

\section{Competing interests}

The authors declare that they have no competing interests.

\section{Publisher's Note}

Springer Nature remains neutral with regard to jurisdictional claims in published maps and institutional affiliations.

\section{Author details}

'Department of Hepatobiliary and Pancreatic Surgery, Gunma University Graduate School of Medicine, Gunma University, 3-39-15 Showa-Machi, Maebashi, Gunma 371-8511, Japan. ${ }^{2}$ Department of Surgery, Fujioka General Hospital, Fujioka, Gunma, Japan. ${ }^{3}$ Department of Pathology, Fujioka General Hospital, Fujioka, Gunma, Japan. ${ }^{4}$ Department of Pathology and

Microbiology, Saga University, Saga, Japan.

Received: 2 August 2018 Accepted: 4 September 2018

Published online: 27 September 2018

\section{References}

1. Wan X, Shi J, Wang A, Xie Y, Yang X, Zhu C, et al. Gallbladder papillary neoplasms share pathological features with intraductal papillary neoplasm of the bile duct. Oncotarget. 2017;8(19):31532-9.

2. Albores-Saavedra J, Adsay NV, Crawford JM, Klimstra DS, Kloppel G, Sripa B, Tsui WMS, Paradis V. Carcinoma of the gallbladder and extrahepatic ducts. In: Bosman FT, Carneiro F, Hruban RH, Theise ND, editors. WHO classification of tumours of the digestive system. 4th ed. Lyon: International Agency for Research on Cancer; 2010. p. 266-73.

3. Ohtsuka M, Shimizu H, Kato A, Yoshitomi H, Furukawa K, Tsuyuguchi T, et al. Intraductal papillary neoplasms of the bile duct. Int J Hepatol. 2014; 2014:459091.

4. Natov NS, Horton LC, Hegde SR. Successful endoscopic treatment of an intraductal papillary neoplasm of the bile duct. World J Gastrointest Endosc. 2017;9(5):238-42.

5. Bennett S, Marginean EC, Paquin-Gobeil M, Wasserman J, Weaver J Mimeault R, et al. Clinical and pathological features of intraductal papillary neoplasm of the biliary tract and gallbladder. HPB (Oxford) 2015;17(9):811-8. 
6. Park SY, Roh SJ, Kim YN, Kim SZ, Park HS, Jang KY, et al. Expression of MUC1, MUC2, MUC5AC and MUC6 in cholangiocarcinoma: prognostic impact. Oncol Rep. 2009;22(3):649-57.

7. Adsay V, Jang KT, Roa JC, Dursun N, Ohike N, Bagci P, et al. Intracholecystic papillary-tubular neoplasms (ICPN) of the gallbladder (neoplastic polyps, adenomas, and papillary neoplasms that are $\geq 1.0 \mathrm{~cm}$ ): clinicopathologic and immunohistochemical analysis of 123 cases. Am J Surg Pathol. 2012; 36(9):1279-301.

8. Sato $R$, Ando $T$, Tateno H, Rikiyama T, Furukawa T, Ebina N. Intracystic papillary neoplasm with an associated mucinous adenocarcinoma arising in Rokitansky-Aschoff sinus of the gallbladder. Surg Case Rep. 2016;2(1):62.

Submit your manuscript to a SpringerOpen ${ }^{\odot}$ journal and benefit from:

- Convenient online submission

- Rigorous peer review

- Open access: articles freely available online

High visibility within the field

- Retaining the copyright to your article

Submit your next manuscript at $\boldsymbol{\nabla}$ springeropen.com 